\title{
Unipolar inductor model coupled to GW emission: energy budget and model application to RX J0806+15 and RX J1914+24
}

\author{
S. Dall'Osso, G. L. Israel, and L. Stella
}

INAF - Osservatorio Astronomico di Roma, via Frascati 33, 00040 Monteporzio Catone (Roma), Italy

e-mail: [dallosso; gianluca; stella]@mporzio.astro.it

Received 16 January 2006 / Accepted 6 November 2006

\section{ABSTRACT}

\begin{abstract}
We further discuss the Unipolar Inductor Model (UIM) coupled to GW emission (Dall'Osso et al. 2006, A\&A, 447, 785) and compare it to observed properties of the two candidate ultrashort period binaries RX J0806+15 and RX J1914+24.

We consider the measured orbital periods, period derivatives and inferred X-ray luminosities of these two sources and find constraints on system parameters in order for the model to account for them. We find that these properties point to the two sources being in different regimes of the UIM, with the requirement of low magnetic moment primaries $\left(\sim 10^{30} \mathrm{G} \mathrm{cm}^{3}\right)$ for both.

Given this weak magnetization, RX J0806+15 has a sufficiently low luminosity that it can be interpreted as having a primary spin almost synchronous to and just slightly slower than the orbital motion. Its measured orbital spin-up is only slightly affected by spinorbit coupling and is mostly due to GW emission.

RX J1914+24, on the other hand, is too bright in X-rays and has too slow an orbital spin-up for the same regime to apply. We suggest that this binary system may be emitting GWs at a significantly higher rate than implied by its measured $\dot{\omega}_{\mathrm{o}} \simeq 6 \times 10^{-17} \mathrm{rad} \mathrm{s}^{-2}$. The latter is explained, in this framework, by the primary spin being slightly faster than the orbital motion $(\alpha \leq 1.1)$. In this case, the associated spin-orbit coupling transfers to the orbit a significant amount of angular momentum, thus partially balancing that lost to GW emission.
\end{abstract}

All expectations can be tested in the near future to confirm the viability of the model.

Key words. gravitational waves - magnetic fields - binaries: close - white dwarfs - X-rays: individuals: RX J0806+15 X-rays: individuals: RX J1914+24

\section{Introduction}

The nature of the two X-ray sources RX J1914+24 and RX J0806+15 has been long debated since their discoveries and the origin of their emission is still quite unclear. Several models have been proposed so far, none of which seems able to explain all of their observed properties without facing major difficulties (see Cropper et al. 2003, for a review on models and observations).

The long-term timing analyses of Strohmayer (2004, 2005), Hakala et al. (2003, 2004) and Israel et al. (2004), the most recent ones based on $\sim 10$ yrs of monitoring, have unambiguously shown that the single periods found in the power-spectra of both objects (respectively $\sim 9.5$ and $5.3 \mathrm{~min}$ ) are steadily decreasing over time. The very nature of such periodicities is debated, evidence being presently in favour of its interpretation as the orbital period of a binary system. Indeed, no other significant periods are found in power spectra which is quite unexpected if, for example, the observed periods reflected the spins of accreting compact objects.

If the observed periods are true orbital modulations, then it is extremely difficult to accomodate Roche-lobe filling nondegenerate secondary stars orbiting a compact companion in such close binary systems, because of the extremely small masses implied. For this reason, double-degenerate binary systems represent the most likely option.

As orbital periods are observed to decrease, accretion models in a double degenerate system are apparently ruled out because (conservative) accretion in these cases should lead to a period increase. Therefore, although with the above caveats, the double-degenerate scenario with no mass exchange seems at least more suited than others to explain the timing properties of these sources.

In this context the unipolar inductor model (UIM), originally proposed for the Jupiter-Io system (Goldreich \& Lynden-Bell 1969), has been suggested as an alternative explanation to the observational properties of the above mentioned sources (Wu et al. 2002). In this version of the model two white dwarfs form a close binary system with a moderately magnetized primary and a secondary that, for practical purposes, is non-magnetic. If the primary spin $\left(\omega_{1}\right)$ is not perfectly synchronous with the orbital motion $\omega_{0}$ (the secondary is tidally locked to the orbit) an e.m.f. is induced across the secondary star, idealized as a perfect conductor crossing the primary's magnetic field lines. In the presence of free charges, the e.m.f. induces a current flow between the two stars, along the sides of the flux tube connecting them. Currents in the atmosphere of the primary cross field lines at a depth where the electrical conductivity $(\sigma)$ becomes isotropical; at this point, they close the circuit returning back to the secondary (Goldreich \& Lynden-Bell 1969; Li et al. 1998; Wu et al. 2002).

Resistive dissipation of currents and the associated heating, that occur essentially in the primary atmosphere, cause the observed soft X-ray emission. The source of the emission is ultimately represented by the mechanical energy of the relative motion between the primary spin and the orbit. Therefore, 
dissipation consumes this energy and brings the primary and the orbit into synchronous rotation.

As shown by Dall'Osso et al. (2006, Paper I from here on), perfect synchronization is never reached in the UIM applied to ultrashort period DDBs. The emission of GWs continuously injects energy in the electric circuit when $\omega_{1}<\omega_{0}$ and can thus sustain a permanent, slight asynchronism of the primary spin. We called this the system's steady-state asynchronism: binaries in this regime, i.e. with sufficiently short orbital periods, will have a primary spin just slightly slower than the orbital motion, the small asynchronism causing a continuous flow of electric current which is ultimately powered by GWs.

In this work, we first address and clarify some general issues discussed in Paper I. Then, we apply our model to the two candidate ultrashort period binaries RX J0806+15 and RX J1914+24. We obtain simple relations between a source luminosity, its measured rate of orbital spin-up, component masses and primary magnetic moment, that give constraints on system parameters for the model to be applicable to both sources.

Detailed and systematic calculations and evolutionary implications are beyond the scope of this work and will be addressed elsewhere.

\section{The Unipolar Inductor Model: summary}

We summarize here briefly the features of the UIM, referring to Wu et al. (2002) and Paper I for a more detailed description. The key ingredient of the model is that the primary spin $\left(\omega_{1}\right)$ differs from the orbital motion $\left(\omega_{0}\right) ; \alpha=\omega_{1} / \omega_{0}$ is the asynchronism parameter.

In an asynchronous system with orbital separation $a$ the secondary star moves across the primary magnetic field lines with a relative velocity $v=a\left(\omega_{0}-\omega_{1}\right)$. The electric field induced through the secondary is $\boldsymbol{E}=\frac{\boldsymbol{v} \times \boldsymbol{B}}{c}$. The associated e.m.f. is $\Phi=2 R_{2} E, R_{2}$ being the secondary's radius. The induced flow of currents is resistively dissipated essentially in the primary atmosphere, causing significant local heating and thus powering the soft X-ray emission. The Lorentz torque on cross-field currents in the primary atmosphere and in the secondary causes spinorbit coupling, redistributing angular momentum between the primary spin and the orbital motion. However, given the significant GW-emission expected from two white dwarfs with ultrashort orbital period, orbital angular momentum is continuously lost from the system, which in turn requires spin-orbit coupling to continue: this keeps the electric circuit active at all times.

Concerning the specific assumptions of this work, we consider binary systems hosting two degenerate white dwarfs, with the following mass-radius relation (Nauenberg 1972):

$$
\frac{R}{R_{\odot}}=0.0112\left[\left(\frac{M}{1.433}\right)^{-\frac{2}{3}}-\left(\frac{M}{1.433}\right)^{\frac{2}{3}}\right]^{\frac{1}{2}}
$$

where $M$ is expressed in solar masses.

In Paper I we have shown that tidal synchronization of the primary component is not expected to be efficient on the orbital evolutionary timescale, while it should well be efficient for a lower mass companion, in most cases of interest. The system orbital evolution is thus given by (Eq. (E4) in Appendix E of Wu et al. 2002):

$$
\frac{\dot{\omega}_{\mathrm{o}}}{\omega_{\mathrm{o}}}=\frac{1}{g\left(\omega_{\mathrm{o}}\right)}\left(\dot{E}_{\mathrm{gr}}-\frac{W}{1-\alpha}\right)
$$

where $\dot{E}_{\mathrm{gr}}=-(32 / 5) G / c^{5}[q /(1+q)]^{2} M_{1}^{2} a^{4} \omega_{\mathrm{o}}^{6}$ is the energy loss rate through GW emission (Landau \& Lifshitz 1951), the second term in parentheses represents the contribution of spin-coupling and $W$ is the electric current dissipation rate (source luminosity). The function $g\left(\omega_{\mathrm{o}}\right)<0$ (see Appendix for its definition) represents two thirds of the orbital (kinetic plus gravitational) energy of the binary system, times a coefficient just slightly smaller than unity. The latter accounts for the additional tiny amount of orbital angular momentum that is continuously lost to keep the secondary into synchronous rotation.

GWs give by definition a positive contribution to $\dot{\omega}_{\mathrm{o}}$ while the contribution of electric coupling ( $W>0$ by definition) depends on the sign of $(1-\alpha)$. Given the measured orbital spin-up of both sources under study, it must be assumed for them that, if $\alpha>1$, then $\left|\dot{E}_{\mathrm{gr}}\right|>|W /(1-\alpha)|$.

The quantity $W$ can be expressed as (Paper I):

$$
\begin{aligned}
W & =\frac{\phi^{2}}{\Re}=\left(\frac{\mu_{1}}{c}\right)^{2} \frac{2 \bar{\sigma} R_{1}^{3 / 2} R_{2}^{3}}{\left[G M_{1}(1+q)\right]^{11 / 6}} \frac{\omega_{\mathrm{o}}^{17 / 3}(1-\alpha)^{2}}{(H / \Delta d) J(e)} \\
& =k \omega_{\mathrm{o}}^{\frac{17}{3}}(1-\alpha)^{2}
\end{aligned}
$$

where $\mathfrak{R}$ is the system effective resistance (see Wu et al. 2002), $\mu_{1}$ the primary's magnetic moment, $\bar{\sigma}$ the height-averaged WD atmospheric conductivity, $H$ the atmospheric depth at which currents cross magnetic field lines and return back to the secondary and $\Delta d$ is the thickness of the arc-like cross section of the current layer at the primary atmosphere. The last equality defines the system's constant $k$.

Finally, we recall the evolution equation for $\alpha$ (see Paper I):

$\frac{\dot{\alpha}}{\alpha}=\frac{W}{\alpha(1-\alpha) I_{1} \omega_{\mathrm{o}}^{2}}-\frac{\dot{\omega}_{\mathrm{o}}}{\omega_{\mathrm{o}}}$,

where $\tau_{\alpha} \equiv \alpha / \dot{\alpha}$.

\section{UIM coupled to GW emission: energy budget and the general solution}

In Paper I we discussed the conditions that cause the current flow and, thus, the soft X-ray emission, never to be quenched by synchronization of the primary star. In the time-independent, linear approximation, the evolution equation for $\alpha$ is solved by a simple exponential function, whose asymptotic value $\alpha_{\infty} \leq 1$ confirms this expectation.

Numerical integration of the full evolution equation confirms that, as the orbital period changes over time, $\alpha$ does indeed evolve asymptotically towards unity, never reaching it exactly. We showed that, in the general solution, the value $\alpha_{\infty}^{\text {en }} \leq 1$ is attained, which conserves the total energy resevoir $\left(E_{\mathrm{UIM}}\right)$ of the circuit

$E_{\mathrm{UIM}}=(1 / 2) I_{1}\left(\omega_{1}^{2}-\omega_{\mathrm{o}}^{2}\right)$.

Therefore, by requiring that the time derivative of $E_{\mathrm{UIM}}$ is zero, we obtain (Paper I)

$\alpha_{\infty}^{\mathrm{en}}\left(1-\alpha_{\infty}^{\mathrm{en}}\right)=\frac{I_{1}}{k} \frac{\dot{\omega}_{\mathrm{o}} / \omega_{\mathrm{o}}}{\omega_{\mathrm{o}}^{11 / 3}}$

This expression for $\alpha_{\infty}^{\text {en }}$ is implicit, as it depends on the source's actual value of $\dot{\omega}_{\mathrm{o}}$, a function of $\alpha$ itself $^{1}$. Despite this, the above formula is of great practical use as it relates the model parameter $\alpha_{\infty}^{\text {en }}$ to measured quantities $\left(\omega_{\mathrm{o}}, \dot{\omega}_{\mathrm{o}}\right)$. We will return on this in Sect. 4.

Given the importance of the steady-state solution, it is necessary to specify here its physical meaning and definition, starting from a discussion of the overall energy budget of the system.

\footnotetext{
1 The problem has a non-linear nature.
} 


\subsection{Asynchronism parameter and efficiency of spin-orbit coupling}

As a preliminary step, we summarize the relevant properties of spin-orbit coupling in the UIM. First recall the expression of the Lorentz torque $N_{\mathrm{L}}=W /\left[\omega_{0}(1-\alpha)\right]$ (Paper I). This same torque $N_{\mathrm{L}}$ acts - with opposite sign - on both the primary star and the orbit. Therefore, angular momentum is conserved by spin-orbit coupling: all that is subtracted from one component is transferred to the other one.

The rate of work done by $N_{\mathrm{L}}$ on the orbit is

$\dot{E}_{\mathrm{L}}^{(\text {orb })}=-N_{\mathrm{L}} \omega_{\mathrm{o}}=-\frac{W}{1-\alpha}$,

while that done on the primary spin is:

$\dot{E}_{\text {spin }}=N_{\mathrm{L}} \omega_{1}=\frac{\alpha}{1-\alpha} W=-\alpha \dot{E}_{\mathrm{L}}^{(\text {orb })}$.

The sum $\dot{E}_{\text {spin }}+\dot{E}_{\mathrm{L}}^{(\text {orb })}=-W$, which means that not all of the energy extracted from one component is transferred to the other one. The energy lost to ohmic dissipation is the energetic cost of spin-orbit coupling. The following picture thus results from the above formulae:

- when $\alpha>1$ the circuit is powered at the expenses of the primary's spin energy. Only a fraction $\alpha^{-1}$ of this energy is transferred to the orbit, the rest being lost to ohmic dissipation;

- when $\alpha<1$ the circuit is powered at the expenses of the orbital energy. Now $\alpha$ measures directly the fraction of energy that is transferred to the primary spin, while $(1-\alpha)$ is that lost to ohmic dissipation ${ }^{2}$.

Therefore, the parameter $\alpha$ can be seen as a measure of the efficiency with which spin-orbit coupling transfers energy between the orbit and the primary spin: the more asynchronous a system is, the less efficiently energy is transferred, most of it being dissipated as heat.

\subsection{Stationary state and the general solution}

We can now re-discuss and clarify the energetic argument of Paper I, based on which we obtained the expression of $\alpha_{\infty}^{\text {en }}$. The steady-state regime is characterized by having a constant electric energy reservoir in the circuit, for a given $\omega_{0}$. The degree of asynchronism is thus set by the condition that the system uses up just the power fed to the circuit by GWs and spin-orbit coupling. It is important to note, in this context, that both ohmic dissipation and the energy required to change $\omega_{0}$ and/or $\omega_{1}$ must be taken into account. Therefore, the condition $\dot{E}_{\mathrm{UIM}}=0$ of Paper I would not be wholly consistent, because only $\dot{\omega}_{1}$ and $\dot{\omega}_{\mathrm{o}}$ appear in $\dot{E}_{\text {UIM }}$ and no account is taken of the luminosity $W$. We then re-state the problem of steady-state in the following terms: since the circuit dissipates energy at a rate $W$, it must recharge at the same rate as the binary evolves, if its energy reservoir is to be conserved. The proper steady-state condition is thus

$\dot{E}_{\mathrm{UIM}}= \pm W$,

where the sign on the right-hand side depends on the asynchronism parameter. The plus sign holds when $E_{\mathrm{UIM}}>0$ and, thus, $\alpha>1$, while the minus sign is relevant to the case with $\alpha<1$ $\left(E_{\mathrm{UIM}}<0\right)$.

\footnotetext{
${ }^{2}$ Note that, for $\alpha=0, \dot{E}_{\text {spin }}=0$ but $\ddot{E}_{\text {spin }}>0$.
}

In Paper I (Appendix B) we have shown that the case with $\alpha_{\infty}>1$ corresponds to a condition very hardly realized in WD+WD systems, because it can hold only for very close systems. So close, indeed, that the lighter component would most likely fill its Roche-lobe. Even if it did not, however, the condition also requires $\dot{\omega}_{\mathrm{o}}<0$, which means the system must widen; the two components will rapidly be too far apart for the condition to hold. Therefore, proper steady-state exists in WD+WD systems only with $\dot{E}_{\mathrm{UIM}}=-W$.

Inserting the expressions for $\dot{\omega}_{1}$ and $W$ in condition (9) we obtain the expression for the steady-state asynchronsim. We call it simply $\alpha_{\infty}$, to distinguish it from the (slightly different) expression of $\alpha_{\infty}^{\text {en }}$ of Paper I.

$$
1-\alpha_{\infty}=\frac{I_{1}}{k} \frac{\dot{\omega}_{\mathrm{o}} / \omega_{\mathrm{o}}}{\omega_{\mathrm{o}}^{11 / 3}}=A .
$$

Our former assumption $\dot{E}_{\text {UIM }}=0$ amounts ultimately to neglecting $W$ compared to the energy terms contained in $\dot{E}_{\mathrm{UIM}}$. Note that the luminosity $W$ is produced by ohmic dissipation, while $I_{1} \omega_{1} \dot{\omega}_{1}=\dot{E}_{\text {spin }}=W \alpha /(1-\alpha)$ becomes much larger than that, the more $\alpha$ gets close to unity. Therefore, neglect of $W$ is actually justified when $\alpha$ is close to 1 , a condition expected to hold in steady-state. Indeed, the expression of Eq. (10) for $1-\alpha_{\infty}$ gives a correction $\sim 1 \%$ to (6), for typical cases of interest.

\section{Model application: equations of practical use}

Equation (10) allows to relate directly the model parameter (1 $\left.\alpha_{\infty}\right)$ to measured quantities, in view of the application of the model to real sources. By contrast, a purely numerical treatment would require solving the evolution equation for $\alpha$ with given (unknown) initial conditions (asynchronism, orbital period, component masses and primary magnetic moment), deriving all other quantities as functions of this solution and comparing to observations.

We have used this latter approach to calculate $\left(1-\alpha_{\infty}\right)$ in the numerical solution for $(1-\alpha)$ : it turns out that, once the system reaches the appropriate regime, the two quantities are essentially coincident. Their ratio at sufficiently short orbital periods $^{3}$ is $\sim 1.001-1.002$, decreasing with the orbital period down to $P \sim 100 \mathrm{~s}$ or even less. Therefore, $\left(1-\alpha_{\infty}\right)$ represents the actual degree of asynchronism of systems with sufficiently short orbital period. It can thus be used to obtain physical constraints on the two systems directly from measured quantities.

\subsection{The asynchronism evolution timescale}

We have shown that the value $\alpha_{\infty}$ (and, accordingly, all steadystate quantities) can be determined in full generality from energetic considerations and can be related to measured quantities and fundamental system parameters (as described below). The evolution of a system in a short-lived, highly asynchronous regime, can be determined only from the integration of the evolutionary equation. In this case, indeed, the free-energy reservoir is represented by the (unknown) primary's degree of asynchronism.

In Paper I we have studied the evolution equation of $\alpha$ in the linear approximation, with constant coefficients. This corresponds to the regime where spin-orbit coupling gives just a negligible contribution to the orbital evolution and, at the same time, the synchronization timescale $\tau_{\alpha}$ is $\ll \tau_{\mathrm{o}}$. A system with

\footnotetext{
${ }^{3}$ Typically $\sim 1000 \mathrm{~s}$, see the definition of $P_{\text {fast }}$ in the next section.
} 
an initial arbitrary value of $\alpha$ may not be in the linear regime: however, the UIM always tends to bring the primary into almost synchronous rotation, so that the linear regime will eventually apply. At this point, in order for the condition $\tau_{\alpha} \ll \tau_{\mathrm{o}}$ to hold, $\left(1-\alpha_{\infty}\right)$ must be sufficiently small or, equivalently, the orbital period sufficiently short. As shown in Paper I, this regime is formally reached for $1-\alpha_{\infty}<3 \times 10^{-2}$ or, equivalently, when the orbital period is shorter than the critical period:

$$
\begin{aligned}
P_{\text {fast }} \simeq & 1.2 \times 10^{3} \mathrm{~s}\left(\frac{M_{1}}{0.9 M_{\odot}}\right)^{-7 / 2}\left(\frac{\mu_{1}}{2.5 \times 10^{30}}\right)^{2} \\
& \times\left(\frac{R_{1}}{6 \times 10^{8}}\right)^{3 / 2}\left(\frac{R_{2}}{1.7 \times 10^{9}}\right)^{3} \frac{\left(\frac{I_{1}}{2.8 \times 10^{50}}\right)^{-1}}{q(1+q)^{3 / 2}\left(\frac{H}{\Delta d}\right) J(e)}
\end{aligned}
$$

where scaling factors are in c.g.s. units. $P_{\text {fast }}$ measures the period below which, in the numerical solution, $(1-\alpha)$ is very well approximated by $\left(1-\alpha_{\infty}\right)$.

Strictly speaking, then, if the orbital period is below the limiting value $P_{\text {fast }}$ the linear, time-independent approximation applies and $\alpha$ reaches $\alpha_{\infty}$ on a time shorter than $\sim 0.03$ times the orbital evolutionary timescale. The probability of catching a system during the short-lived, asynchronous phase is accordingly very small. Most systems with $P<P_{\text {fast }}$ are caught in the longlived steady-state.

We finally note that, when $\alpha$ is very close to 1 , it can change only by a factor $\ll \alpha$. Therefore, the timescale $\tau_{\alpha}=\alpha / \dot{\alpha}$ can become very long just because of this. However, as $\alpha$ passes from 1.1 to 1.001 , changing by $\sim 10 \%$ only, $(1-\alpha)$ decreases by two orders of magnitude and the same holds for $\dot{E}_{\mathrm{L}}^{(\text {orb })}$, while the dissipation rate $W$ changes by four orders of magnitude.

In this situation, the evolution timescale of $1-\alpha$ provides more direct information on the evolving properties of the system, because this parameter evolves towards zero. We thus define, neglecting the minus sign in the last equality:

$$
\begin{aligned}
\tau_{(1-\alpha)} & =\frac{1-\alpha}{d(1-\alpha) / d t}=-\frac{1-\alpha}{\alpha} \tau_{\alpha} \\
& =\left(1-\alpha_{\infty}\right) \tau_{\mathrm{o}}\left[1-\alpha \frac{1-\alpha_{\infty}}{1-\alpha}\right]^{-1} .
\end{aligned}
$$

The difference between this and $\tau_{\alpha}$ is negligible as long as $\alpha \gg$ 1 and becomes important when $|1-\alpha|<1$. In particular, for $\alpha=\alpha_{\infty}, \tau_{\alpha_{\infty}}=\alpha_{\infty} /\left(1-\alpha_{\infty}\right) \tau_{\mathrm{o}}$, much longer than $\tau_{\mathrm{o}}$. Indeed, $\alpha$ changes only very slightly once it is close to 1 .

On the other hand, $\tau_{\left(1-\alpha_{\infty}\right)}=\tau_{\mathrm{o}}$, from which the basic meaning of steady-state is recovered clearly: it is the regime in which the system's degree of asynchronism evolves on exactly the same timescale of the orbital period.

For any $\alpha \neq \alpha_{\infty}$ the corresponding timescale becomes quickly much shorter than $\tau_{\mathrm{o}}$, thus hampering the possibility of observing systems that are too far from steady-state, as stated above.

\subsection{Relating measurements to system parameters}

We can now summarize the formulae that allow a direct comparison with the measured timing properties and inferred luminosities of the two sources.

The key parameter $\left(1-\alpha_{\infty}\right)$ depends only on the measured quantities $\dot{\omega}_{\mathrm{o}}$ and $\omega_{\mathrm{o}}$ (with no assumption on the relative strength of GW and spin-orbit coupling), on the primary moment of inertia $I_{1}$ and on the unknown parameter $k$, the value of which is primarily determined by $\mu_{1}$. Therefore, based on measurements of $\omega_{0}, \dot{\omega}_{\mathrm{o}}$ and $W$, we can define the quantity $\alpha_{\infty}$ as a function of $k$ and use it to estimate $\mu_{1}$. In particular, given the definition of $\alpha_{\infty}$ and $W$ (Eqs. (10) and (3), respectively), we have

$W=I_{1} \omega_{\mathrm{o}} \dot{\omega}_{\mathrm{o}} \frac{(1-\alpha)^{2}}{1-\alpha_{\infty}}$

The source steady-state luminosity can thus be written as:

$W_{\infty}=I_{1} \dot{\omega}_{0} \omega_{0}\left(1-\alpha_{\infty}\right)$.

The equation for the orbital evolution (2) provides a further relation between the three measured quantities, component masses and degree of asynchronism. We re-write it here in a form that makes the dependence on measured quantitites and unknown parameters explicit:

$\dot{E}_{\mathrm{gr}}-g\left(\omega_{\mathrm{o}}\right)\left(\dot{\omega}_{\mathrm{o}} / \omega_{\mathrm{o}}\right)=\frac{W}{(1-\alpha)}$

that becomes, inserting the appropriate expressions for $\dot{E}_{\mathrm{gr}}$ and $g\left(\omega_{\mathrm{o}}\right)$ (cf. Sect. 2), and neglecting the small correction to $g\left(\omega_{\mathrm{o}}\right)$ due to tidal locking of the secondary:

$\frac{32}{5} \frac{G^{7 / 3}}{c^{5}} \omega_{\mathrm{o}}^{10 / 3} X^{2}-\frac{1}{3} G^{2 / 3} \frac{\dot{\omega}_{\mathrm{o}}}{\omega_{\mathrm{o}}^{1 / 3}} X+\frac{W}{1-\alpha}=0$,

where $X \equiv M_{1}^{5 / 3} q /(1+q)^{1 / 3}=\mathcal{M}^{5 / 3}, \mathcal{M}$ being the system's chirp mass.

With all above equations we can now turn to each of the two systems and assess which region of the UIM parameter space applies to each of them.

\section{RX J0806+15}

Israel et al. (2003) measured an on-phase X-ray luminosity (in the range $0.1-2.5 \mathrm{keV}) L_{\mathrm{X}}=8 \times 10^{31}(d / 200 \mathrm{pc})^{2} \mathrm{erg} \mathrm{s}^{-1}$ for this source. These authors suggested that the bolometric luminosity might even be dominated by the (unseen) value of the UV flux, and reach values 5-6 times higher (i.e. $L_{\text {bol }} \sim$ $\left.5 \times 10^{32} \mathrm{erg} \mathrm{s}^{-1} d_{200}^{2}\right)$. The optical flux is only $\sim 15 \%$ pulsed, indicating that most of it might not be associated to the unipolar inductor mechanism (possibly the cooling luminosity of the white dwarf plays a role). Given these uncertainties and, mainly, the uncertainty in the distance to the source, we assume here a luminosity $W \simeq 10^{32}(d / 200 \mathrm{pc})^{2} \mathrm{erg} \mathrm{s}^{-1}$. In Sect. 7 we consider the effect of assuming a larger source luminosity for RX J0806+15 (and RX J1914+24 as well), and show that conclusions are affected only weakly.

Israel et al. (2004) and Strohmayer (2005) obtained independent, phase-coherent timing solutions for the orbital period of this source over a $\sim 10$ yrs baseline, that are fully consistent within the errors. The solution reported by Israel et al. (2004) is $\dot{P}=-3.67(1) \times 10^{-11}$ and $P=321.53033(2)$. These give $\dot{\omega}_{\mathrm{o}} \simeq 2.23 \times 10^{-15} \mathrm{rad} \mathrm{s}^{-2}$ and $\omega_{\mathrm{o}} \simeq 0.0195 \mathrm{rad} \mathrm{s}^{-1}$.

In Fig. 1 (see caption for further details), the dashed line represents the locus of points in the $M_{2}$ vs. $M_{1}$ plane, for which the measured $\omega_{\mathrm{o}}$ and $\dot{\omega}_{\mathrm{o}}$ are consistent with being due to GW emission only, i.e. if spin-orbit coupling was absent $(\alpha=1)$. This corresponds to a chirp mass $\mathcal{M} \simeq 0.3 M_{\odot}$. 


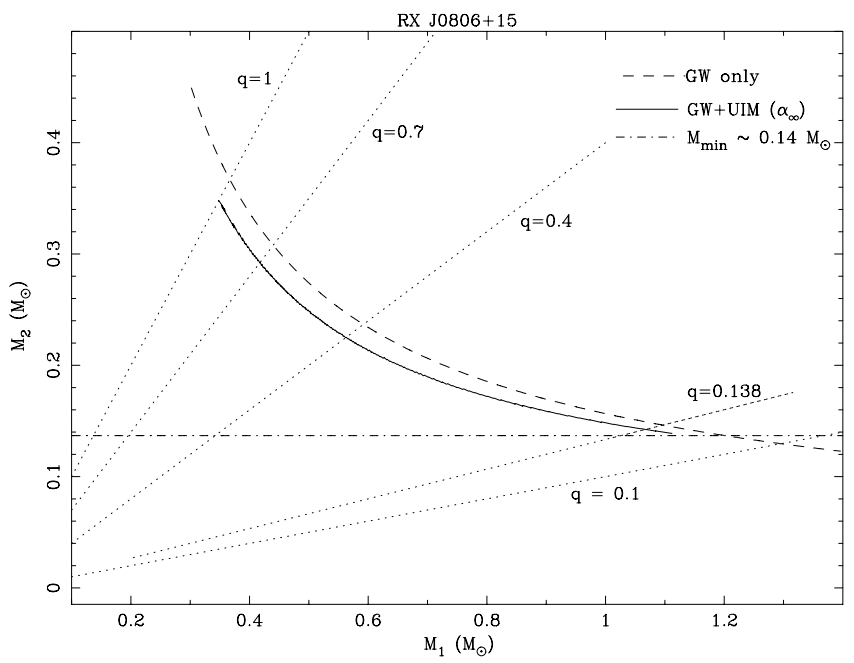

Fig. 1. $M_{2}$ vs. $M_{1}$ plot based on the measured timing properties of RX J0806+15. The dashed curve is the locus expected if orbital decay is driven by GW alone, with no spin-orbit coupling. The solid line describes the locus expected if the system is in a steady-state, with $(1-\alpha)=\left(1-\alpha_{\infty}\right) \simeq 8 \times 10^{-3}$. The horizontal dot-dashed line represents the minimum mass for a degenerate secondary not to fill its Roche-lobe at an orbital period of $321.5 \mathrm{~s}$. Dotted lines are the loci of fixed mass ratio.

Inserting the measured quantities in Eq. (13) and assuming a reference value ${ }^{4}$ of $I_{1}=3 \times 10^{50} \mathrm{~g} \mathrm{~cm}^{2}$, we obtain:

$\frac{(1-\alpha)^{2}}{1-\alpha_{\infty}} \simeq \frac{10^{32} d_{200}^{2}}{1.3 \times 10^{34}} \simeq 8 \times 10^{-3} d_{200}^{2}$.

In principle, the source may be in any regime, but its short orbital period strongly suggests that it is beyond the limiting period $P_{\text {fast }}$ of Eq. (11). Therefore, the most likely and natural scenario is that it has already settled into steady-state and $\alpha=\alpha_{\infty}$.

In this case, we see from Eq. (17) that $\left(1-\alpha_{\infty}\right) \simeq 8 \times 10^{-3}$. Once UIM and spin-orbit coupling are introduced, the locus of allowed points in the $M_{2}$ vs. $M_{1}$ plane is somewhat sensitive to the exact value of $\alpha$ : the solid curve of Fig. 1 was obtained for $\alpha=\alpha_{\infty}=0.992 . M_{1}$ must be smaller than $1.1 M_{\odot}$ in order for the secondary not to fill its Roche lobe, thus avoiding mass transfer. Note that, if $M_{1}>1.2 M_{\odot}, I_{1} \leq 10^{50} \mathrm{~g} \mathrm{~cm}^{2}$ and assuming $W=$ $W_{\infty}$ would imply $1-\alpha_{\infty}>8 \times 10^{-3}$. In this case, the solid curve would be shifted downwards, crossing the minimum-mass horizontal line at $M_{1}<1 M_{\odot}$. In turn this would rule out the high $M_{1}$ values based on which the curve was obtained. Therefore, we conclude that, if RX J0806+15 is interpreted as being in the UIM steady-state, $M_{1} \leq 1 M_{\odot}$ is necessarily required.

From $\left(1-\alpha_{\infty}\right)=8 \times 10^{-3}$ we obtain, by use of Eq. (3), $k \simeq 7.7 \times 10^{45}(\mathrm{cgs})$ : from this, component masses and primary magnetic moment can be constrained (see Fig. 1). Indeed, since $k=\hat{k}\left(\mu_{1}, M_{1}, q ; \bar{\sigma}\right)$ (see its definition in Eq. (3)), we have a further constraint on it, because $M_{1}$ and $q$ must lie along the solid curve of Fig. 1. Given the value of $\bar{\sigma}, \mu_{1}$ is obtained for each point along the solid curve.

According to $\mathrm{Wu}$ et al. (2002), and references therein, the electrical conductivity of a white dwarf atmosphere with a temperature $\sim 10^{5} \mathrm{~K}$ has a value $\sim 10^{13} \div 10^{14}$ (esu). We take $\bar{\sigma}=$ $3 \times 10^{13}$ (esu), so that the whole range above is within a factor 3 .

${ }^{4}$ Possible values of $I_{1}$ range in a limited interval: $I_{1}=3.6 \times$ $10^{50} \mathrm{~g} \mathrm{~cm}^{2}$ for $M=0.2 M_{\odot}, I_{1}=3.75 \times 10^{50} \mathrm{~g} \mathrm{~cm}^{2}$ for $M=0.5 M_{\odot}$, $I_{1}=3.2 \times 10^{50} \mathrm{~g} \mathrm{~cm}^{2}$ for $M=0.8 M_{\odot}, I_{1}=2.4 \times 10^{50} \mathrm{~g} \mathrm{~cm}^{2}$ if $M_{1}=1 M_{\odot}$ and $I_{1} \simeq 10^{50} \mathrm{~g} \mathrm{~cm}^{2}$ for $M_{1}=1.3 M_{\odot}$.

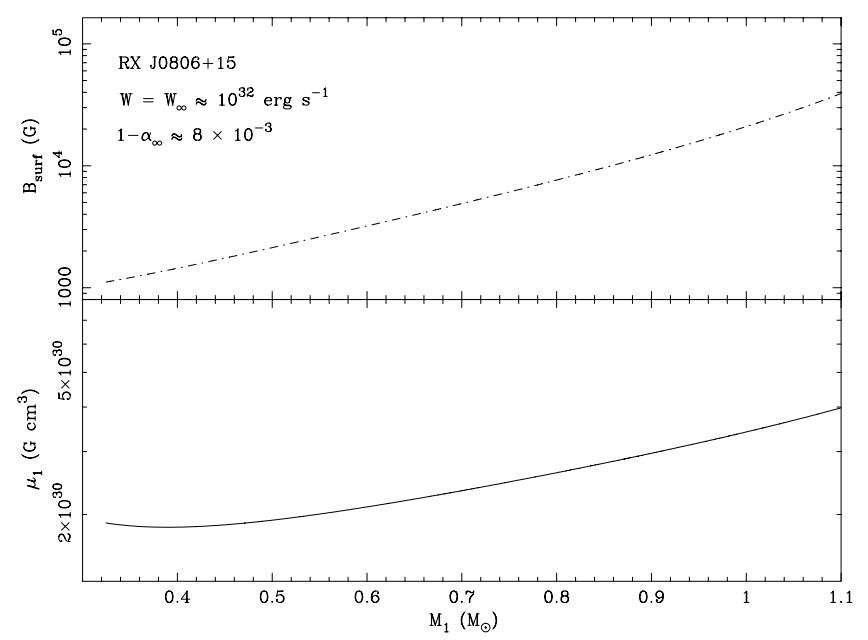

Fig. 2. The value of the primary magnetic moment $\mu_{1}$, and the corresponding surface $B$-field, as a function of the primary mass $M_{1}$, for $(1-\alpha)=\left(1-\alpha_{\infty}\right)=8 \times 10^{-3}$.

The values of $\mu_{1}$ obtained in this way, and the corresponding field at the primary's surface, are plotted in Fig. 2, from which $\mu_{1} \sim$ a few $\times 10^{30} \mathrm{G} \mathrm{cm}^{3}$ results, somewhat sensitive to the primary mass.

We note further that the solid curve of Fig. 1 is not strictly a locus of constant chirp mass. However, it is nearly so: $X \simeq$ $(3.4 \div 4.5) \times 10^{54} \mathrm{~g}^{5 / 3}$ along it, which implies $\mathcal{M} \simeq(0.26 \div$ $0.31) M_{\odot}$. More importantly, $\dot{E}_{\mathrm{gr}} \simeq(1.1 \div 1.9) \times 10^{35} \mathrm{erg} \mathrm{s}^{-1}$ and, since $W /\left(1-\alpha_{\infty}\right)=\dot{E}_{\mathrm{L}}^{\text {(orb) }} \simeq 1.25 \times 10^{34} \mathrm{erg} \mathrm{s}^{-1}$, we have $\dot{E}_{\mathrm{gr}} \simeq(9 \div 15) \dot{E}_{\mathrm{L}}^{\text {(orb) }}$. Therefore, orbital spin-up is essentially driven by $\mathrm{GW}$ alone; indeed, the dashed and solid curves are very close on the $M_{2}$ vs. $M_{1}$ plane.

Summarizing, we have shown that RX J0806+15 has observational properties that can be well interpreted in the UIM framework, assuming it is in steady-state. This requires the primary to be moderately magnetic, with $\mu_{1} \sim 10^{30} \mathrm{G} \mathrm{cm}^{3}$ and with an almost synchronous spin, slightly slower than the orbital motion (the difference being less than $\sim 1 \%$ ). The small degree of asynchronism is consistent with the fact that no modulation of pulse arrival times at the beat period $\omega_{\mathrm{b}}=\omega_{\mathrm{o}}-\omega_{1}$ is observed, since its small amplitude would likely be below the intrinsic timing noise of the source (cf. Barros et al. 2004). The expected value of the magnetic moment can also be tested by future observations, for example through studies of polarized emission, at optical and/or radio wavelengths (Willes \& Wu 2004).

\section{RX J1914+24}

The luminosity of this source has been subject to many revisions over the last few years. Wu et al. (2002) refer to earlier ASCA measurements that, for a distance of $200-500 \mathrm{pc}$, corresponded to a luminosity in the range $\left(4 \times 10^{33} \div 2.5 \times 10^{34}\right) \mathrm{erg} \mathrm{s}^{-1}$. Ramsay et al. (2005), based on more recent XMM-Newton observations and a standard blackbody fit to the X-ray spectrum, derived an $\mathrm{X}$-ray luminosity of $\simeq 10^{35} d_{\mathrm{kpc}}^{2} \mathrm{erg} \mathrm{s}^{-1}$, where $d_{\mathrm{kpc}}$ is the distance in kpc. The larger distance of $\sim 1 \mathrm{kpc}$ was based on a work by Steeghs et al. (2006). Still more recent XMM-Newton observtions have been analyzed by Ramsay et al. (2006): these authors find that an optically thin, thermal emission spectrum, with an edge at $0.83 \mathrm{keV}$ (attributed to O VIII) gives a significantly better fit to the data than the previously used blackbody model. Compared to a blackbody, the optically thin thermal plasma 
model clearly leads to a source bolometric luminosity much lower than previously derived, $L_{\mathrm{bol}} \simeq 10^{33} d_{\mathrm{kpc}}^{2} \mathrm{erg} \mathrm{s}^{-1}$. Ramsay et al. (2006) also note that the determination of a $1 \mathrm{kpc}$ distance is not free of uncertainties and that a much smaller distance $(\sim 200 \mathrm{pc})$ is still possible: based on the latter, the luminosity would become as small as $\sim 3 \times 10^{31} \mathrm{erg} \mathrm{s}^{-1}$.

Given these large discrepancies, interpretation of this source's properties is clearly ambiguous and quite dependent on which assumptions are made. We refer here to the more recent assessment by Ramsay et al. (2006) of a luminosity $L=10^{33} \mathrm{erg} \mathrm{s}^{-1}$ for a $1 \mathrm{kpc}$ distance, but want to stress mainly the general characteristics of our procedure. The numerical estimates that we obtain are, at this stage, necessarily illustrative and, in Sect. 7, we show how derived quantities change when different luminosities are assumed.

Ramsay et al. (2006) also find possible evidence, at least in a few observations, of two secondary peaks in power spectra. These are very close to $\left(\Delta v \simeq 5 \times 10^{-5} \mathrm{~Hz}\right)$ and symmetrically distributed around the strongest peak at $\sim 1.76 \times 10^{-3} \mathrm{~Hz}$, the latter usually interpreted as the orbital period of the source. We will briefly comment on these possible features at the end of Sect. 6.3.

Ramsay et al. (2005), confirmed by Ramsay et al. (2006), also give the most up to date measurement of the source's rate of orbital evolution, $\dot{P}-3.2(10) \times 10^{-12}$, that converts to $\dot{\omega}_{\mathrm{o}} \simeq 6.2 \times 10^{-17} \mathrm{rad} \mathrm{s}^{-2}$ for the orbital period of $569 \mathrm{~s}$, or $\omega_{\mathrm{o}} \simeq 0.011 \mathrm{rad} \mathrm{s}^{-1}$. We warn that these parameters were not obtained, like in the case of RX J0806+15, through a phasecoherent analysis, therefore they may be subject to larger uncertainties and future revision.

Application of the scheme used for RX J0806+15 to this source is not as straightforward, since its properties indicate that it is not in a steady-state and, likely, not even in the regime where spin-orbit coupling is negligible in the orbital evolution.

First of all, its inferred luminosity seems inconsistent with being associated to steady-state as, with the measured values of $\omega_{\mathrm{o}}$ and $\dot{\omega}_{\mathrm{o}}$, Eq. (13) gives ${ }^{5}$

$$
\begin{aligned}
\frac{(1-\alpha)^{2}}{1-\alpha_{\infty}} & =\frac{10^{33}}{2 \times 10^{32}} d_{\mathrm{kpc}}^{2} \\
& \simeq 5 d_{\mathrm{kpc}}^{2},
\end{aligned}
$$

which has important implications. First of all, given $\omega_{\mathrm{o}}$ and $\dot{\omega}_{\mathrm{o}}$, the system steady-state luminosity should be $\ll 2 \times 10^{32} \mathrm{erg} \mathrm{s}^{-1}$ (Eq. (14)), hardly consistent with measurements. Indeed, even if a large value of $\left(1-\alpha_{\infty}\right) \simeq 10^{-1}$ was assumed, $W \simeq 2 \times$ $10^{31} \mathrm{erg} \mathrm{s}^{-1}$, less than the smallest possible luminosity reported by Ramsay et al. (2006). A relatively high ratio between the actual asynchronism parameter and its steady-state value appears unavoidable. Indeed, from the above equation:

$|1-\alpha| \simeq 2.2\left(1-\alpha_{\infty}\right)^{1 / 2}$.

For example, if this system had the same $k$ and $I_{1}$ as RX J0806+15, it would have a 2.5 times smaller value of $\left(1-\alpha_{\infty}\right) \simeq 3.3 \times 10^{-3}$, from which $|1-\alpha| \simeq 0.13$.

\subsection{The case for $\alpha>1$}

The low rate of orbital shrinking implied by the measured $\dot{\omega}_{\mathrm{o}} \sim$ $6.2 \times 10^{-17} \mathrm{~s}^{-2}$ (Ramsay et al. 2005) suggests a very low mass system, with $M_{1} \leq 0.3 M_{\odot}$; in this case the secondary would not

\footnotetext{
5 Again assuming $I_{1}=3 \times 10^{50} \mathrm{~g} \mathrm{~cm}^{2}$.
}

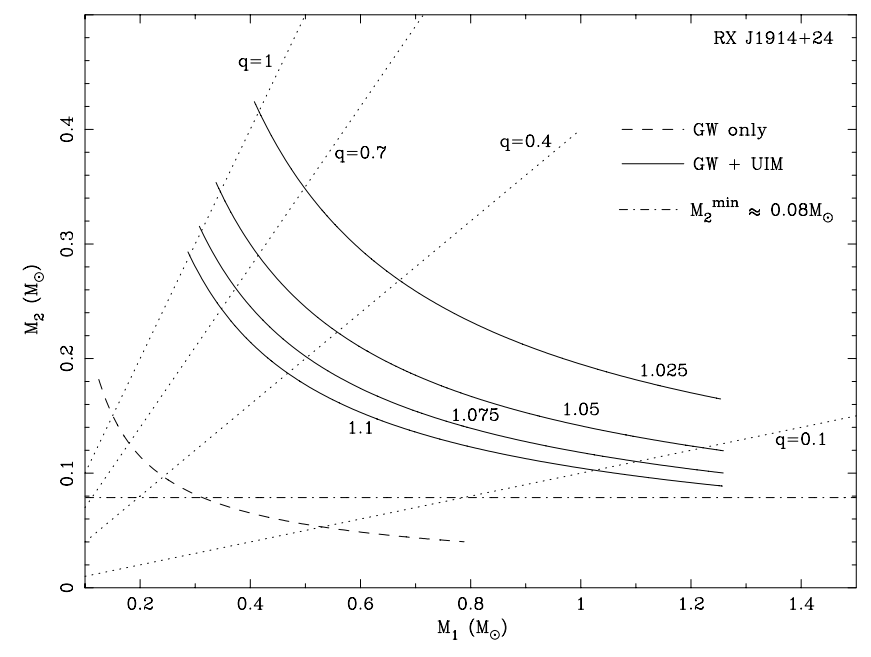

Fig. 3. $M_{2}$ vs. $M_{1}$ plot based on measured timing properties of RX J1914+24. The dot-dashed line corresponds to the minimum mass for a degenerate secondary not to fill its Roche-lobe. The dashed curve represents the locus expected if orbital decay was driven by GW alone, with no spin-orbit. coupling. It is seen that this curve is consistent with a detached system only if both masses are extremely low. The solid lines describe the loci expected if spin-orbit coupling for the primary star (the secondary spin is tidally locked to the orbit) is present and gives a negative contribution to $\dot{\omega}_{0}$. The four curves are obtained assuming $W=10^{33} \mathrm{erg} \mathrm{s}^{-1}$ and four different values of $\alpha=1.025,1.05,1.075$, 1.1 , respectively, from top to bottom, as reported in the plot.

fill its Roche-lobe only for $q \geq 0.26$ (see Fig. 3 and Nelemans 2004 , for a discussion of this point). In the UIM framework, this result gives important hints as to the primary spin.

If, as shown above, the system is far from steady-state and has $\alpha<1$, the locus of allowed masses in the $M_{2}$ vs. $M_{1}$ plane is shifted downwards with respect to the dashed line of Fig. 3 by a significant amount. This would exacerbate the problem of the very low component masses. In particular because, $\alpha$ being quite different from 1, the downward shift of the curve in the $M_{2}$ vs. $M_{1}$ plane would accordingly be large.

This argument can be made more cogent by using the measured value of $\dot{\omega}_{\mathrm{o}}>0$; a strong constraint on $|1-\alpha|$ results, because the high inferred luminosity $W \sim 10^{33} \mathrm{erg} \mathrm{s}^{-1}$ would imply a high value of $\dot{E}_{\mathrm{L}}^{\text {(orb) }}$. If $\alpha<1$, this latter term sums to the GW torque: the resulting orbital evolution would thus be faster than if it were driven by GW alone. In fact, the smallest possible value of $\dot{E}_{\mathrm{L}}^{\text {(orb) }}$ for $\alpha<1$ is obtained with $\alpha=0$, from which $\dot{E}_{\mathrm{L}}^{(\mathrm{orb})}=10^{33} \mathrm{erg} \mathrm{s}^{-1}$.

Therefore, if $\alpha<1$, an absolute minimum to the system's rate of orbital shrinking would result from the above minimum rate of orbital energy extraction operated by spin-orbit coupling. This minimum $\dot{\omega}_{\mathrm{o}}$ turns out to be very close to the measured one, so that it would imply unplausibly small component masses in order for $\dot{E}_{\mathrm{gr}}$ to be sufficiently small. This can be shown by comparing the corresponding chirp masses. The dashed curve in Fig. 3 corresponds to a value of $X \simeq 1.07 \times 10^{54} \mathrm{~g}^{5 / 3}$, or a chirp mass $\mathcal{M} \simeq 0.13 M_{\odot}$. On the other hand, an equivalent GW luminosity of $10^{33} \mathrm{erg} \mathrm{s}^{-1}$ would correspond to $X \simeq 8.5 \times$ $10^{53} \mathrm{~g}^{5 / 3}$ or an equivalent chirp mass $\mathcal{M} \simeq 0.12 M_{\odot}$.

Therefore, the slow measured rate of orbital evolution of this source essentially rules out the case $\alpha<1$ in the UIM framework discussed here: indeed, no point in the $M_{2}$ vs. $M_{1}$ plane can be found for $\alpha<1$ if a non-Roche-lobe-filling secondary is required (as it is in the UIM). We note that the absolute 
minimum is obtained assuming $\alpha=0$, a very unlikely condition. Summarizing, Fig. 3 and the above argument strongly lead to consider the case where the primary spin is faster than the orbital motion. This would indeed offer a great advantage in interpreting this source: in the case $\alpha>1$, spin-orbit coupling has an opposite sign with respect to the GW torque. The two mechanisms have opposite effects on the orbital evolution and a slow measured $\dot{\omega}_{\mathrm{o}}$ can result from the two opposite torques partially cancelling each other.

We want to stress further the above arguments, as there appears to be some confusion in the literature. Marsh \& Nelemans (2005) have recently claimed that, in order for the UIM to account for the properties of RX J1914+24, $\alpha \geq 10^{2}$ would be required, implying the primary is spinning nearly at break-up.

These authors arrive at their conclusion first deriving an estimate of the GW luminosity from the measured $\omega_{\mathrm{o}}$ and $\dot{\omega}_{\mathrm{o}}$ and then requiring that $W /(1-\alpha)$ be less than the estimated $\dot{E}_{\mathrm{gr}}$. Given the high source luminosity (they assume $10^{35} \mathrm{erg} \mathrm{s}^{-1}$ ) and small $\dot{\omega}_{\mathrm{o}}$, a large value of $|1-\alpha|$ is required in order for that condition to hold. Note that, assuming $W \simeq 10^{33} \mathrm{erg} \mathrm{s}^{-1}$, their argument gives $|1-\alpha|>1$, not so extreme indeed. Most importantly, however, this argument relies on the assumption that the measured timing parameters of this source give a reliable estimate of its actual GW luminosity. This assumption holds only if spin-orbit coupling is negligible; we have shown above that this needs not be the case and, most likely, is not the case in this source. The argument by Marsh \& Nelemans might thus be reversed and used as evidence that a non-negligible spin-orbit coupling is necessary in applications of the UIM to this source.

Ramsay et al. (2006) suggest that the much smaller luminosity of $10^{33} \mathrm{erg} \mathrm{s}^{-1}$ they derive would weaken the objection by Marsh \& Nelemans, since it would require an asynchronism $(1-\alpha) \sim 10^{-3}$ only (or even less). It is easy to see that this is not true, because with $W=10^{33} \mathrm{erg} \mathrm{s}^{-1}$ and $(1-\alpha) \leq 10^{-3}$ one would obtain $\dot{E}_{\mathrm{L}}^{\text {(orb) }}=W /(1-\alpha) \geq 10^{36} \mathrm{erg} \mathrm{s}^{-1}$, which is greater than any plausible GW emission from this system, given its measured $\dot{\omega}_{0}$. Rather, $|1-\alpha|$ cannot be too small without making the model inconsistent with the observed slow orbital spin-up.

The conclusion of this section can only be that, in a selfconsistently constructed UIM framework, the GW luminosity of this source has to be larger than implied by $\dot{\omega}_{\mathrm{o}}$, which implies $\alpha>1$, otherwise the model hardly applies.

\subsection{Constraining the asynchronous system}

We try now to give a quantitative description of the possible scenario introduced above. The goal is to find constraints on system parameters in order to match the measured luminosity and $\dot{\omega}_{\mathrm{o}} / \omega_{\mathrm{o}}$ and meet the requirement that the resulting regime has a sufficiently long lifetime.

Given that it is not possible to determine all system parameters uniquely, the scheme that we have used is as follows: given a value of $\alpha$, the measured $\dot{\omega}_{\mathrm{o}}, \omega_{\mathrm{o}}$ and $W$ allow us to determine $\dot{\alpha}$ from Eq. (4). For each value of $M_{1}$ Eq. (2), re-written in the form reported in the Appendix, can thus be used to determine the value of $M_{2}$ (or $q$ ) that is compatible with $\dot{\omega}_{\mathrm{o}}$ and $\omega_{\mathrm{o}}$. This yields the solid curves of Fig. 3.

As these curves show, the larger is $\alpha$ and the smaller the upward shift of the corresponding locus, which may seem surprising. However, these curves are obtained at fixed luminosity $W$ and $\dot{\omega}_{\mathrm{o}}$. Recalling that $(1 / \alpha)$ gives the efficiency of angular momentum transfer in systems with $\alpha>1$ (cf. Sect. 3.1), a higher $\alpha$ at a given luminosity implies that less energy and angular

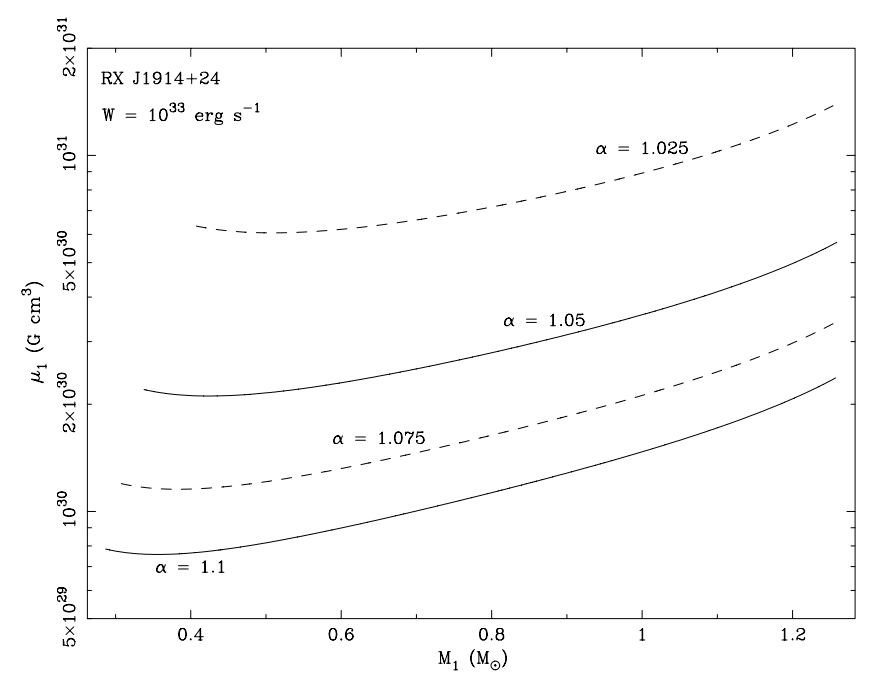

Fig. 4. $\mu_{1}$ as a function of the primary mass for the same values of $\alpha$ used previously and reported on the corresponding curves. Given a value of $\alpha$ and the estimated luminosity $W \sim 10^{33} \mathrm{erg} \mathrm{s}^{-1}, \dot{\alpha}$ is calculated from Eq. (4) as a function of $M_{1} . q$ as well is obtained as a function of $M_{1}$ from Fig. 3. All system parameters contained in $k$ are thus determined, apart from $\mu_{1}$. The estimated luminosity $W$ and the assumed value of $\alpha$ give the corresponding value of $k$ : combining all the information, $\mu_{1}$ is self-consistently determined.

momentum are being transferred to the orbit. Accordingly, GWs must be emitted at a smaller rate for a given $\dot{\omega}_{0}$.

The values of $\alpha$ in Fig. 3 were chosen arbitrarily and are just illustrative: note that the resulting curves are quite similar to those obtained for RX J0806+15. Given $\alpha$, one can also estimate $k$ from the definiton of $W$ (Eq. (3)). On the other hand, the information given by the curves of Fig. 3 yields determination of all quantities contained in $k$, apart from $\mu_{1}$. Therefore, assuming $\bar{\sigma}=3 \times 10^{13}$ (esu) as in the previous case, we can determine the value of $\mu_{1}$ along each of the four curves of Fig. 3. Derived values are plotted in Fig. 4 . We see that $\mu_{1}$ varies by just a factor 2-3 as a function of $M_{1}$ along each curve, while it depends somewhat more strongly on the assumed value of $\alpha$.

The resulting numbers correspond in any case to a moderately magnetic primary, indeed quite similar to what obtained for RX J0806+15 (the surface field $B$ is in the $10^{2} \div 10^{5} \mathrm{G}$ range, depending on the primary mass).

\subsection{Lifetime of the asynchronous state}

Finally, having argued that this system can be explained in the UIM framework by a relatively highly asynchronous and moderately magnetic system, we have to deal with the timescale problem.

While RX J0806+15 is fully consistent with being in steadystate, so that it has no timescale problem, we have demonstrated that RX J1914+24 cannot be in the same regime. Moreover, given the combination of a high luminosity and slow orbital evolution, we have found arguments in favour of its primary spinning faster than the orbital motion. It must be fast enough, indeed, that the orbital evolution is significantly slowed down by the angular momentum transferred to the orbit by spin-orbit coupling. Therefore, the system is in the fully non-linear regime where spin-orbit coupling is non negligible and the evolution equation for $\omega_{\mathrm{o}}$ must be solved explicitly.

We can estimate the synchronization timescale $\tau_{\alpha}$ starting from the evolution Eq. (4). With the measured values of $W, \omega_{0}$ 


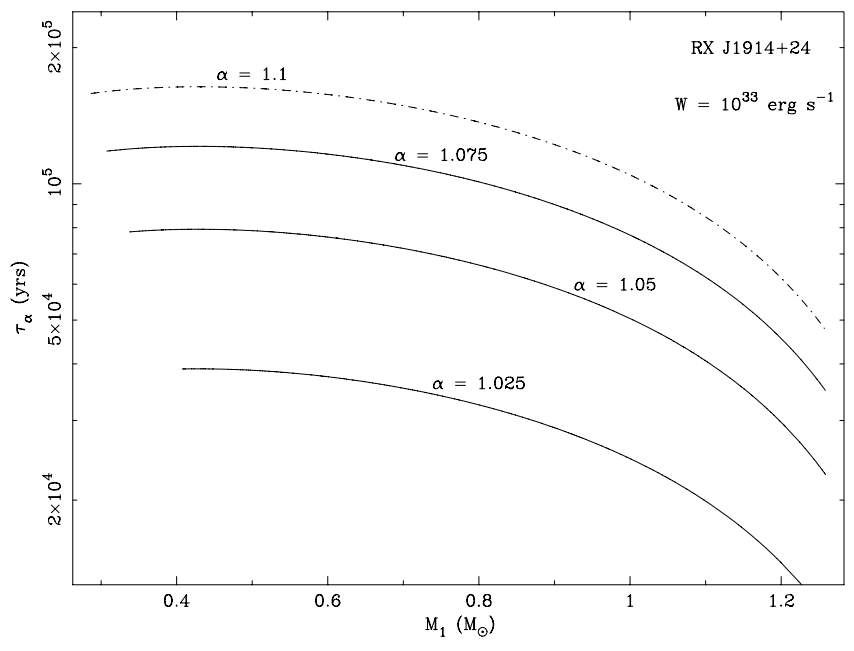

Fig. 5. The evolution timescale $\tau_{\alpha}$ as a function of the primary mass for the same values of $\alpha$ used previously, reported on the corresponding curves. Given the estimated luminosity $W \sim 10^{33} \mathrm{erg} \mathrm{s}^{-1}$ and a value of $\alpha, \tau_{\alpha}$ is calculated from Eq. (4) as a function of $I_{1}$ and, hence, $M_{1}$.

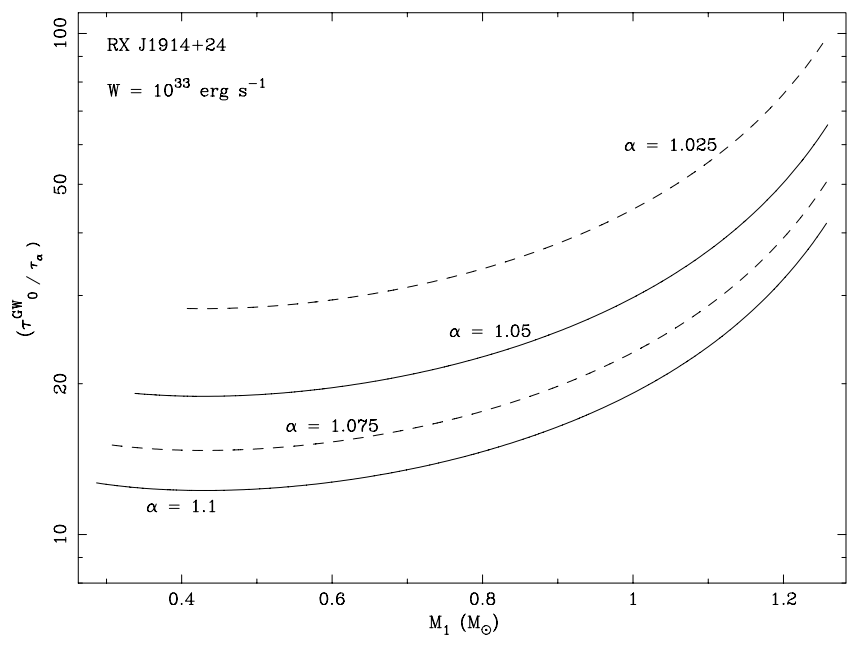

Fig. 6. The ratio $\tau_{\mathrm{o}}^{\mathrm{GW}} / \tau_{\alpha}$ calculated for the four values of $\alpha=$ $1.025,1.05,1.075,1.1 . \tau_{\mathrm{o}}^{\mathrm{GW}}$ is obtained from the value of $X$ along each of the four curves of Fig. 3, according to Eq. (20), while the corresponding value of $\tau_{\alpha}$ are those plotted in Fig. 5.

and $\dot{\omega}_{0}, \tau_{\alpha}$ can be calculated as a function of $I_{1}$ (and, thus, of $M_{1}$ ) given a particular value of $\alpha$. In Fig. 5 we have reported the results obtained for the same four values of $\alpha$ assumed in the previous section.

It is apparent that the larger $\alpha$, the longer the timescale, for a given value of $M_{1}$. This is because the system luminosity is a fixed (measured) quantity: therefore the larger the asynchronism, the smaller the efficiency of spin-orbit coupling and, accordingly, the larger $\tau_{\alpha}$. The resulting timescales range from a few $\times 10^{4}$ yrs to a few $\times 10^{5}$ yrs, while $\tau_{\mathrm{o}} \sim 6 \times 10^{6} \mathrm{yrs}$ for this source. Although $\tau_{\alpha}$ may still seem too short, we stress that in this scenario the long orbital evolutionary timescale results largely from the effect of spin-orbit coupling. The latter decreases strongly on the timescale $\tau_{\alpha}$, after which $\tau_{\mathrm{o}}$ is essentially determined by GW emission only. We denote the latter as $\tau_{\mathrm{O}}^{\mathrm{GW}}$. Therefore, the system will actually spend the total time $\tau_{\mathrm{o}}^{\mathrm{GW}}$ at the nearly constant orbital period, its evolution being slowed down by just a fraction $\tau_{\alpha} / \tau_{\mathrm{o}}^{\mathrm{GW}}$ of this time. In Fig. 6 we have plotted the ratio $\left(\tau_{\mathrm{o}}^{\mathrm{GW}} / \tau_{\alpha}\right)$ for the four cases studied above
(Figs. 3-5). The values $\tau_{\mathrm{o}}^{\mathrm{GW}}$ were calculated along the solid curves of Fig. 3, that define the value of $X$ for each $\left(M_{1}, M_{2}\right)$. Given $X$, both the source GW luminosity $\dot{E}_{\mathrm{gr}}$ and $\tau_{\mathrm{o}}^{\mathrm{GW}}$ can be calculated. In particular, since $g\left(\omega_{\mathrm{o}}\right)$ is a function of $X$ itself, we have

$\left(\frac{\dot{\omega}_{\mathrm{o}}}{\omega_{\mathrm{o}}}\right)^{\mathrm{GW}}=\left(\tau_{\mathrm{o}}^{\mathrm{GW}}\right)^{-1}=\frac{96}{5} \frac{G^{5 / 3}}{c^{5}} \omega_{\mathrm{o}}^{8 / 3} X$

According to the above reasoning, the expected GW luminosity of this source is in the range $(4.6 \div 1.4) \times 10^{34} \mathrm{erg} \mathrm{s}^{-1}$. The corresponding ratios $\dot{E}_{\mathrm{gr}} / \dot{E}_{\mathrm{L}}^{\text {(orb) }}$ are $1.15,1.21,1.29$ and 1.4 , respectively, for $\alpha=1.025,1.05,1.075$ and 1.1.

Therefore, RX J1914+24 and RX J0806+15 may even have similar component masses and magnetic moments. The significant observational differences would essentially be due to the two systems being caught in different evolutionary stages. RX J1914+24 would be in a luminous, transient phase that preceeds its settling into the dimmer steady-state, a regime already reached by the shorter period RX J0806+15. Although the more luminous phase is transient, its lifetime can be as long as $\sim 10^{5} \mathrm{yrs}$, one or two orders of magnitude longer than previously estimated.

We comment here on the two symmetric peaks, around the main one, found in a few power spectra by Ramsay et al. (2006).

In the UIM framework, given a primary spin $\omega_{1}$ that differs from $\omega_{0}$, some signature of both periodicities may be expected, indeed. The hot spot position on the primary surface is modulated at the orbital period (the spot always follows the position of the secondary star) and at the beat period $\omega_{\mathrm{b}}=\omega_{1}-\omega_{\mathrm{o}}$. The latter because the spot position is determined by the position of the primary's magnetic pole as well, which itself rotates at $\omega_{1}$. If the field simmetry axis were perfectly aligned to the spin (and orbital) axis, this second modulation would disappear but, in general, some inclination is to be expected. Barros et al. (2004) have shown that, in the context of the UIM, the modulation at $\omega_{\mathrm{b}}$ in RX J1914+24 is small, possibly negligible, if the inclination angle $\beta<(15-19)^{\circ}$. However, it would be a natural expectation of the model to have two main periodicities in power spectra, namely $\omega_{\mathrm{o}}$ and $\omega_{\mathrm{b}}$ : when $\omega_{1} \sim \omega_{\mathrm{o}}$ (thus $\alpha \sim 1$ ) the frequency $\omega_{\mathrm{b}} \ll \omega_{\mathrm{o}}$.

This is highly speculative at this stage and proper quantitative studies of this phenomenon, both on the observational and theoretical side, are necessary before these features can be used to put quantitative constraints on models. These are beyond the scope of this paper and are currently in progress. What we want to stress here is that a second periodicity in power spectra is not a finding against the UIM. The features reported by Ramsay et al. (2006) are not in constrast with qualitative expectations in the UIM.

\section{Dependence of the results on the assumed luminosity}

In this section we briefly discuss how our results change when different source luminosities are assumed, reflecting the large uncertainties in their distances and bolometric emission. We have repeated the same reasoning presented in the previous sections, simply changing the adopted value of $W$ to a significantly larger value. 


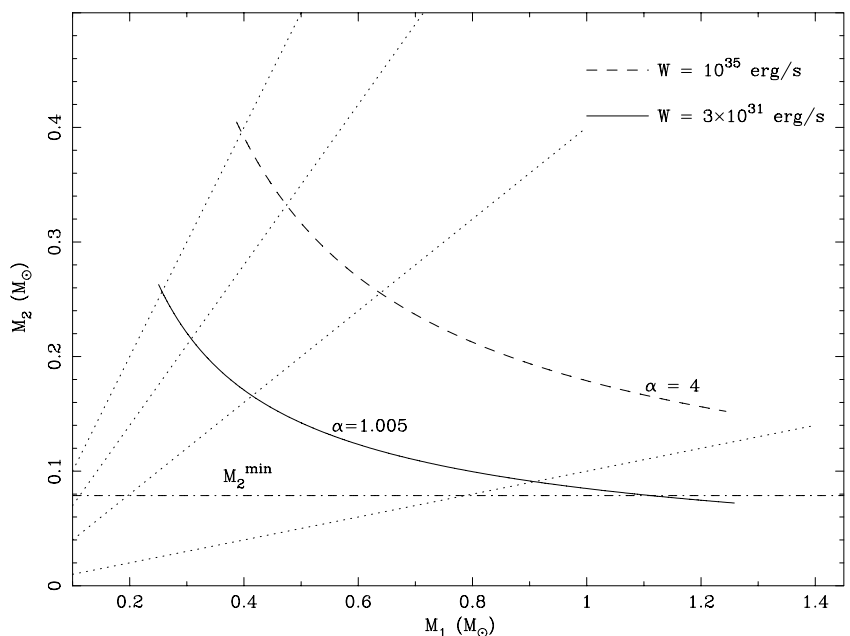

Fig. 7. The loci in the $M_{2}$ vs. $M_{1}$ plane obtained assuming a luminosity of $W=3 \times 10^{31} \mathrm{erg} / \mathrm{s}$ and $\alpha=1.005$ (solid line) or a very high luminosity of $\sim 10^{35} \mathrm{erg} / \mathrm{s}$ and $\alpha=4$ (dashed line). As noted in the previous section, curves shift upwards for smaller values of $\alpha$ and downwards for increasing values of the asynchronism.

\section{1. $R \times J 0806+15$}

We have discussed in Sect. 5 the uncertainty in the bolometric luminosity of this source, which could possibly be up to a factor 5-6 larger than the X-ray emission reported by Israel et al. (2003). We assume here $W=5 \times 10^{32} \mathrm{erg} \mathrm{s}^{-1}$ and see how our conclusions change.

For this luminosity, a six times larger degree of asynchronism than previously assumed would result, $1-\alpha_{\infty} \simeq 3.8 \times 10^{-2}$, still a quite small number. Given this value, the corresponding locus in the $M_{2}$ vs. $M_{1}$ plane would correspond to values of $X$ and, accordingly, GW luminosities smaller by just a few percent. The corresponding primary magnetic moment and implied surface field are themselves decreased by the same factor of six by which $W$ increases. All these changes, although non-negligible on quantitative grounds, do not affect significantly the overall picture presented above.

\section{2. $R X J 1914+24$}

The case of RX J1914+24 is more difficult: the luminosity assumed in Sect. 6 lies at the centre of a very wide range of proposed values, from the smallest $W \simeq 3 \times 10^{31} \mathrm{erg} \mathrm{s}^{-1}$ (Ramsay et al. 2006) to the largest $W \simeq 10^{35} \mathrm{erg} \mathrm{s}^{-1}$ (Ramsay et al. 2005). We show and briefly discuss here the same figures presented in the previous section, obtained for just the two extreme luminosities above. Instead of exploring a range of values of $\alpha$, we are exploring the range of values of $W$, over approximately four orders of magnitude. Therefore just one curve, is reported for each of the two cases. In all figures below the solid lines correspond to the low luminosity $W=3 \times 10^{31} \mathrm{erg} \mathrm{s}^{-1}$, while the dashed curves represent the opposite case with $W=10^{35} \mathrm{erg} \mathrm{s}^{-1}$. From Fig. 7 we see that the high luminosity case would require a significant degree of asynchronism, but still of the order of a few, not the $\gg 100$ value previously suggested in the literature. On the other hand, the very dim case can be well described by a low asynchronism, even lower than the steady-state asynchronism found for RX J0806+15. However, we have shown that $1-\alpha_{\infty} \simeq 0.15$ would be implied for this source if $3 \times 10^{31} \mathrm{erg} \mathrm{s}^{-1}=W_{\infty}$. Rather, $\alpha=1.005$ gives $\left(1-\alpha_{\infty}\right) \simeq 1.7 \times 10^{-4}$ (Eq. (19)).

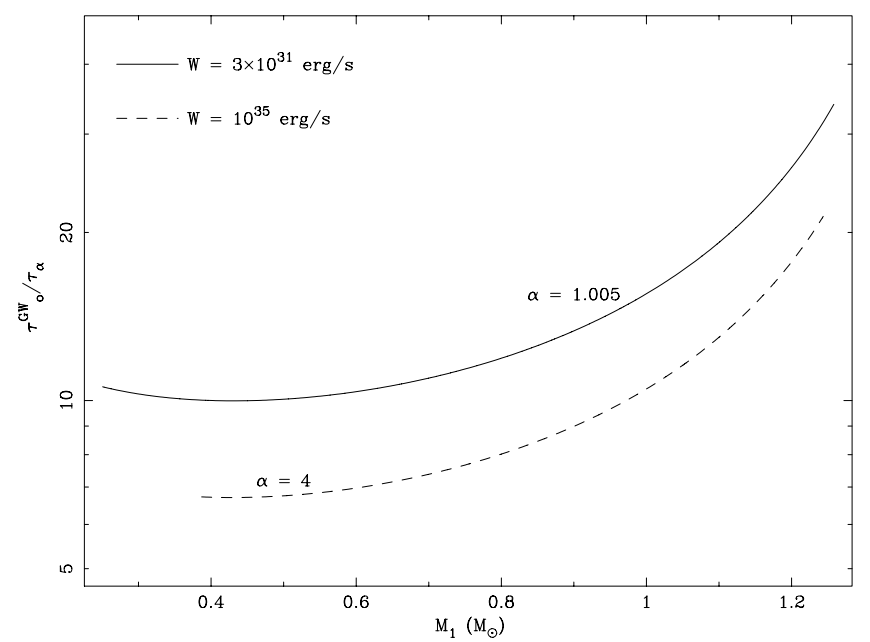

Fig. 8. The ratio $\tau_{\mathrm{o}}^{\mathrm{GW}} / \tau_{\alpha}$ calculated for $W=3 \times 10^{31} \mathrm{erg} / \mathrm{s}$ and $\alpha=1.005$ (solid line) and for $\mathrm{W}=10^{35} \mathrm{erg} / \mathrm{s}$ and $\alpha=4$ (dashed line). $\tau_{\mathrm{o}}^{\mathrm{GW}}$ is obtained from the value of $X$ along the corresponding curve of Fig. 7, according to Eq. (20), while the corresponding value of $\tau_{\alpha}$ are calculated as described in the caption to Fig. 5.

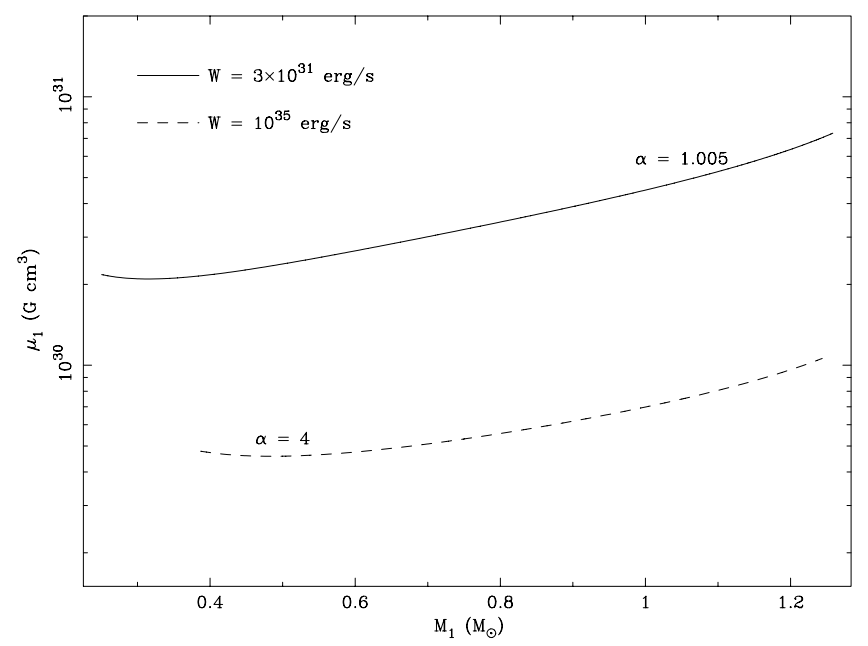

Fig. 9. The expected value of the primar's magnetic moment $\mu_{1}$ as a function of the primary mass $\left(M_{1}\right)$ for the same two extreme luminosities of the above figures, indicated in the figure. Despite the non negligible difference between the two curves, these still span the range of medium-to-weak magnetization already found previously.

The dimmer system would have a long lifetime, $\tau_{\alpha} \sim 3 \times$ $10^{5} \mathrm{yrs}$, which is $\sim$ one tenth to one twentieth of the orbital evolutionary timescale $\tau_{\mathrm{o}}^{\mathrm{GW}}$. From the solid curve of Fig. 7 we can estimate a GW luminosity $\dot{E}_{\mathrm{gr}} \simeq 10^{34} \mathrm{erg} \mathrm{s}^{-1}$ and a ratio $\dot{E}_{\mathrm{gr}} / \dot{E}_{\mathrm{L}}^{(\text {orb })} \sim 1.6$. On the other hand, the very luminous system has a shorter $\tau_{\alpha} \sim$ a few $\times 10^{4}$ yrs, but can still have a lifetime $\sim$ one tenth of the orbital evolutionary timescale $\tau_{\mathrm{o}}^{\mathrm{GW}}$. The latter is itself significantly shorter than $\omega_{0} / \dot{\omega}_{0}$, because large component masses would be required and, correspondingly, very strong GW emission expected. For the case with $\alpha=4$ we estimate $\dot{E}_{\text {gr }} \simeq 3.6 \times 10^{34} \mathrm{erg} \mathrm{s}^{-1}$, with a ratio $\sim 1.25$ to the rate of work $W /(1-\alpha)$.

Finally, Fig. 9 shows the expected primary magnetic moments in both cases. 


\section{Conclusions}

In the present work we have clarified some key aspects of spin-orbit coupling in the unipolar inductor model coupled to GW and applied the model to the two candidate ultrashort period binaries RX J0806+15 and RX J1914+24. We derive constraints on system parameters in order for the model to account for the inferred luminosities and measured $\omega_{0}$ and $\dot{\omega}_{\mathrm{o}}$ of both sources. The UIM accounts in a natural way for their luminosities, measured orbital periods and rates of orbital spinup, provided they have moderately magnetic primaries, say $\mu_{1} \sim$ several $\times 10^{29}$ to few $\times 10^{30} \mathrm{G} \mathrm{cm}^{3}$. Concerning each of the sources, we conclude that:

- In the case of RX J0806+15 the observations are explained in the framework of the steady-state solution. The degree of asynchronism is determined by a balance between the rate of current dissipation and the rate at which energy is fed to the electrical circuit by orbital evolution, while the source luminosity does not decrease at all. The orbital evolution is driven mostly by GW-emission and the system's timing properties should differ only slightly from those of two point masses evolving under GW emission. The modest primary magnetic moment represents a key requirement of this interpretation and its measurement would be a crucial test as to its viability.

- RX J1914+24, on the other hand, cannot have reached the steady-state solution yet, as its present rate of electric energy dissipation is significantly higher than what GW emission alone could feed to the circuit.

In addition to requiring a moderately magnetic primary (again $\mu_{1} \sim 10^{30} \mathrm{Gcm}^{3}$ ), we conclude that the primary spin frequency must be somewhat larger than the orbital frequency. For $W \simeq 10^{33} \mathrm{erg} \mathrm{s}^{-1}$ the parameter $(1-\alpha)$ can be in the range $(0.1 \div 0.02)\left(P_{\text {spin }}\right.$ between 517 and $\left.558 \mathrm{~s}\right)$. Although the source is not consistent with being in a steadystate we have shown that, for $\alpha$ in the above range, the asynchronous, luminous and transient phase can last between a few $\times 10^{4}$ yrs up to $3 \times 10^{5}$ yrs. These are between $2 \%$ and $10 \%$ of the orbital evolution timescale. Therefore, there is a sizeable probability that one in two of the ultrashort period binaries that have so far been found is in this evolutionary stage.

The measured value of $\dot{\omega}_{\mathrm{o}}$ does not reflect the actual rate of GW emission from this system. It rather results from the sum of GWs and spin-orbit coupling, the latter partially cancelling the effect of the former. For the range of values of $\alpha$ that we consider more likely, $\alpha \simeq(1.025 \div 1.1)$, the actual GW luminosity should be in the $\sim 10^{34} \mathrm{erg} \mathrm{s}^{-1}$ range.

Values of $\alpha$ between 1.005 and $\sim 4 \div 5$ can explain all possible source luminosities in the range $\left(3 \times 10^{31} \div 10^{35}\right) \mathrm{erg} \mathrm{s}^{-1}$. In particular, a luminosity as high as $10^{35} \mathrm{erg} \mathrm{s}^{-1}$ can be selfconsistently explained with $\alpha \sim$ of a few, extremely less then previously claimed in the literature.

In this work we were mainly concerned with finding system parameters for which the model could be made consistent with the energetic requirements and timing properties of the candidate sources. Further work is needed to address issues such as the duration and shape of the X-ray pulsations. Predictions that can be checked through observations to be carried out in the next few years are essentially of three kinds:

- independent measurements of the primary magnetic moment $\mu_{1}$, which should result to be smaller than several $\times 10^{30} \mathrm{G} \mathrm{cm}^{3}$ in order for the model to account for the two sources. Although several details of the model should still be taken into account, it seems difficult that the overall energetic requirements could be changed by orders of magnitude.

- extending the temporal baseline over which timing studies are carried out may help detecting the second derivative of $\omega_{\mathrm{o}}$ in both systems, but particularly in RX J1914+24. Such measurements could provide an indipendent estimate of system masses and/or confirm a significant contribution of UI to the orbital evolution;

- a detailed study of the duration and shape of the X-ray pulsations, as well as of power spectra, would be required, in order to put constraints on geometric properties of the systems, possibly related to the shape and orientation of magnetic field lines near the primary component.

Account for hot spot shapes different from a simple filled spot should be taken since two detached, arc-like hot regions are expected instead (see Wu et al. 2002).

Concerning the longer term future, we emphasize that LISA is expected to measure the GW emission of very short period binaries such as those discussed here, therefore yielding an independent check of the model. In particular, RX J1914+24 is expected to be much more luminous in GWs than one would infer based on its measured $\omega_{\mathrm{o}}$ and $\dot{\omega}_{\mathrm{o}}$.

\section{Appendix}

We derive here the expression used to obtain the solid curves of Fig. 3. In the case of Fig. 1, $\alpha$ was assumed to be constant, as this describes the relevant condition to RX J0806+15. On the other hand, in studying RX J1914+24 we were led to deal with the more general case in which $\alpha$ has a non negligibile time derivative.

Let $g\left(\omega_{0}\right)$ be defined as:

$g\left(\omega_{\mathrm{o}}\right)=-\frac{1}{3}\left[\frac{q^{3} G^{2} M_{1}^{5} \omega_{\mathrm{o}}^{2}}{1+q}\right]^{1 / 3}\left[1-\frac{6}{5}(1+q)\left(\frac{R_{2}}{a}\right)^{2}\right]$

where the second term in square brackets is denoted by $f\left(\omega_{0}\right)$. Then, from Eqs. (2), (4) and the definition of $g\left(\omega_{\mathrm{o}}\right)$ :

$\frac{\dot{\omega}_{\mathrm{o}}}{\omega_{\mathrm{o}}}=\frac{1}{g\left(\omega_{\mathrm{o}}\right)}\left[\dot{E}_{\mathrm{gr}}-\left(\frac{\dot{\alpha}}{\alpha}+\frac{\dot{\omega}_{\mathrm{o}}}{\omega_{\mathrm{o}}}\right) \alpha I_{1} \omega_{\mathrm{o}}^{2}\right]$

and from this, re-writing the pure-GW period derivative as $\left(\dot{\omega}_{\mathrm{o}} / \omega_{\mathrm{o}}\right)_{g}=\dot{E}_{\mathrm{gr}} f\left(\omega_{\mathrm{o}}\right) / g\left(\omega_{\mathrm{o}}\right)$ :

$\frac{\dot{\omega}_{\mathrm{o}}}{\omega_{\mathrm{o}}}\left[1+\alpha \frac{I_{1} \omega_{\mathrm{o}}^{2}}{g\left(\omega_{\mathrm{o}}\right)}\right]=\left(\frac{\dot{\omega}_{\mathrm{o}}}{\omega_{\mathrm{o}}}\right)_{g}\left[\frac{1}{f\left(\omega_{\mathrm{o}}\right)}-\frac{\dot{\alpha} I_{1} \omega_{\mathrm{o}}^{2}}{g\left(\omega_{\mathrm{o}}\right)\left(\frac{\dot{\omega}_{\mathrm{o}}}{\omega_{\mathrm{o}}}\right)_{g}}\right]$

that leads to the following expression:

$\frac{\dot{\omega}_{\mathrm{o}}}{\omega_{\mathrm{o}}}=\frac{\left(\dot{\omega}_{\mathrm{o}} / \omega_{\mathrm{o}}\right)_{g}}{g\left(\omega_{\mathrm{o}}\right)+\alpha I_{1} \omega_{\mathrm{o}}^{2}}\left[\frac{g\left(\omega_{\mathrm{o}}\right)}{f\left(\omega_{\mathrm{o}}\right)}-\dot{\alpha} \frac{I_{1} \omega_{\mathrm{o}}^{2}}{\left(\dot{\omega}_{\mathrm{o}} / \omega_{\mathrm{o}}\right)_{g}}\right]$.

Now substitute the expressions for $g\left(\omega_{0}\right)$ and $f\left(\omega_{0}\right)$, remembering that $I_{1}=(2 / 5) M_{1} R_{1}^{2}$ and that, from Kepler's third law, $\omega_{\mathrm{o}}^{-8 / 3}=\left[G M_{1}(1+q)\right]^{-4 / 3} a^{4}$. After some algebra it obtains:

$$
\begin{aligned}
\frac{\dot{\omega}_{\mathrm{o}}}{\omega_{\mathrm{o}}}= & \left(\frac{\dot{\omega}_{\mathrm{o}}}{\omega_{\mathrm{o}}}\right)_{g}\left[1+\frac{\dot{\alpha}}{16 q^{2}}\left(\frac{R_{1}}{a}\right)^{2} \frac{c^{5} a^{4}}{\left(G M_{1}\right)^{3}}\right] \\
& \times\left[1-\frac{6}{5} \alpha \frac{1+q}{q}\left(\frac{R_{1}}{a}\right)^{2}-\frac{6}{5}(1+q)\left(\frac{R_{2}}{a}\right)^{2}\right]^{-1}
\end{aligned}
$$


The solid curves of Fig. 3 were obtained just using Eq. (25). This takes account of the primary asynchronism and its rate of variation as well, while assumes the secondary to be perfectly synchronous (tidally locked) at all times.

\section{References}

Barros, S. S. C., Marsh, T. R., Groot, P., et al. 2004 [arXiv: astro-ph/0412368]

Clarke, J. T., Ballester, G. E., Trauger, J., et al. 1996, Science, 274, 404

Cropper, M., Ramsay, G., Wu, K., \& Hakala, P. 2003 [arXiv: astro-ph/0302240]

Dall'Osso, S., et al. 2006, A\&A, 447, 785

Goldreich, P., \& Lynden-Bell, D. 1969, ApJ, 156, 59

Hakala, P., Ramsay, G., Wu, K., et al. 2003, MNRAS, 343, L10

Hakala, P., Ramsay, G., \& Byckling, K. 2004, MNRAS, 353, 453
Israel, G. L., Covino, S., Stella, L., et al. 2003, ApJ, 598, 492

Israel, G. L., Covino, S., Dall'Osso, S., et al. 2004, Mem. Soc. Astron. It. Suppl., 5,148

Israel, et al. 2005, in preparation

Landau, L., \& Lifshitz, E. 1951, The Classical Theory of Fields, 1st. edn. (Reading, Mass.: Addison-Wesley Publishing Co.)

Li, J., Ferrario, L., \& Wickramashinghe, D. 1998, ApJ, 503, L151

Marsh, T. R., \& Nelemans, G. 2005, MNRAS, 363, 581

Nauenberg, M. 1972, ApJ, 175, 417

Nelemans, G. 2004 [arXiv: astro-ph/0409676]

Norton, A. J., Haswell, C. A., \& Wynn, G. A. 2004, A\&A, 419, 1025

Ramsay, G., Hakala, P., Wu, K., et al. 2005, MNRAS, 357, 49

Ramsay, G., Cropper, M., \& Hakala, P. 2006 [arXiv:astro-ph/0601104]

Strohmayer, T. E. 2002, ApJ, 581, 577

Strohmayer, T. E. 2004, ApJ, 610, 416

Willes, A. J., \& Wu, K. 2004, MNRAS, 348, 285

Wu, K., Cropper, M., Ramsay, G., \& Sekiguchi, K. 2002, MNRAS, 331, 221 